\title{
Studying the fractional derivative for natural convection in slanted cavity containing porous media
}

\author{
Sameh E. Ahmed ${ }^{1,2}$ (D) M. A. Mansour ${ }^{3} \cdot$ E. A-B. Abdel-Salam ${ }^{4} \cdot$ Eman F. Mohamed $^{3}$
}

(c) Springer Nature Switzerland AG 2019

\begin{abstract}
This paper studies the numerical solution of the unsteady free convention in a slanted cavity under effect of the fractional derivative containing a porous medium. To simplify all the computations, the main equations are mapped from the irregular physical domain into a regular polygon in a shape of a rectangular computational domain utilizing nonlinear axis transformations. Using the finite differences method, the fractional partial differential equations are solved. The primary results are teased in the ordinary case at $\alpha=1, R a=1000$ and $\varphi=0.0$ and those are found in an excellent agreement with the previous results from the open literatures. The obtained numerical data are represented in terms of the isotherms and streamlines contours as well as the local and average Nusselt numbers at the heated wall. Wide ranges of the key-parameters are considered i.e. orders of the fractional derivatives $\alpha$ and $\beta$ are varied from 1 to 0.7 , the Rayleigh number Ra is varied from $10^{2}$ to $10^{4}$ and the inclination angle takes the values $\varphi=0^{\circ}, 15^{\circ}, 30^{\circ}$ and $45^{\circ}$. The results revealed that the decrease in order of the fractional derivatives enhances the fluid activity while both of the local and average Nusselt numbers are reduced regardless of the Rayleigh number values.
\end{abstract}

Keywords Natural convection · Slanted enclosures · Porous media · Fractional derivative · Finite difference method (FDM)

\begin{tabular}{|c|c|c|c|}
\hline $\begin{array}{l}\text { List o } \\
a\end{array}$ & $\begin{array}{l}\text { symbols } \\
\text { Cavity width }\end{array}$ & $\begin{array}{l}x, y \\
X, Y\end{array}$ & $\begin{array}{l}\text { Cartesian coordinates } \\
\text { Transmutation coordinates }\end{array}$ \\
\hline$L$ & Cavity length & $D^{\alpha}$ & Operator of the fractional derivatives with respect \\
\hline$t$ & Time & & to Cartesian coordinates $x, y$ \\
\hline$A$ & The ratio between height and width & $D^{\beta}$ & Operator of the fractional derivatives with respect \\
\hline$g$ & Gravity constant & & to time $t$ \\
\hline$q_{w}$ & Wall heat flux & & \\
\hline$K$ & Permeability of the porous medium & $a$ & Fffective thermal diffusivity of the norous medium \\
\hline k & $\begin{array}{l}\text { Effective thermal conductivity of the porous } \\
\text { medium }\end{array}$ & $\begin{array}{l}a \\
\beta\end{array}$ & Coefficient of thermal expansion \\
\hline $\mathrm{Nu}$ & Nusselt number & $\Delta T$ & Temperature difference \\
\hline$N u_{m}$ & Average Nusselt number & $\theta$ & Dimensionless temperature \\
\hline$T$ & Temperature of fluid & $\tau$ & Dimensionless time \\
\hline$T_{c}$ & For cold wall temperature & $\xi, \eta$ & Dimensionless variables \\
\hline$T_{h}$ & For hot wall temperature & & Kinematic viscosity \\
\hline & & $\psi$ & Stream function \\
\hline
\end{tabular}

Sameh E. Ahmed, Sehassan@kku.edu.sa; M. A. Mansour, mansour201354@yahoo.com; E. A-B. Abdel-Salam, emad_abdelsalam@yahoo.com; Eman F. Mohamed, eman.fm@science.au.edu.eg| ${ }^{1}$ Department of Mathematics, Faculty of Science, King Khalid University, Abha, Saudi Arabia. ${ }^{2}$ Mathematics Department, Faculty of Sciences, South Valley University, Qena 83523,

Egypt. ${ }^{3}$ Department of Mathematics, Faculty of Science, Assiut University, Assiut, Egypt. ${ }^{4}$ Department of Mathematics, Faculty of Science, New Valley University, El-Kharja 72511, Egypt. 
$\phi \quad$ Inclination angle

$\Psi \quad$ Dimensionless stream function

$\varepsilon \quad$ Prescribed error

\section{Introduction}

\subsection{Introduction of natural convection}

No one can deny that studying of the natural convection is very important especially in the porous media because it has a lot of application in industrial, engineering and agricultural such as building insulation, grain storage, groundwater decontamination, solar collector, thermal drying process, casting solidification and petroleum reserve, etc. All these applications and other are widely reviewed in [1-6] As a result of this large numbers of applications of this phenomenon, it has become an important research topic in the recent centuries. In general, the researchers focus on investigating of the numerical solution of convection for triangular, square or rectangular enclosures because of it is easy to solve. Varol et al. [7]. At first, they used finite difference method then they applied successive under relaxation method. Temperature diffusion and flow field were discussion at different aspect ratio and Rayleigh numbers. They noted that the location of heater has effect such as when located it at the bottom wall a lot of whirlpool were formed and the highest heat transfer obtained conferring to the of other cases. Mansour and Ahmed [8] studied the free convection heat transfer in a slanted triangular cavity contained nanofluid (Cu-water) in porous media under the effect generation of heat. They found that the average Nusselt number became better when increase the nanoparticle volume fraction also, increasing parameter of heat generation causes to low in the value of the average Nusselt number. A numerical studied of unstable magneto hydrodynamic natural convection in a slanted cavity contain a porous medium with heat generation inside has been performed by Mansour et al. [9]. They studied two cases, the first case considered all wall of enclosure at zero degree of temperature and the second case the vertical walls are kept adiabatic and they presented results of the average Nusselt number for some variables conditions. In 2011, Mansour et al. [10] discussed the problem of double-expanded convection in slanted triangular cavity containing a porous medium with different sinusoidal of the boundary conditions in the being of sink heat source, numerically. They found that the fluid motion is accelerated in case of the being of heat sink and the fluid motion is decayed in the presence of heat source.

\subsection{Brief introduction on fractional calculus}

The fractional calculus attracted a lot of researches in the last and present centuries. The theory of fractional calculus improved as theoretical branch for mathematicians. Recently many papers have been presented in the fractional derivatives rules which aiming to generalize rules of ordinary derivatives. Also, there are a lot of applications of this direction in mechanics, control theory, chemistry and physics, so on $[11,12]$. It was begun in 1695, when L'Hopital asked if the expression $\frac{d^{0.5}}{d x^{0.5}}$ has any meaning. Since then, many researches started to try to replace the concept of the usual derivative to fractional derivatives. The most famous of them, Riemann-Liouville which relied on repeating the integral operator $n$ time and used the famous Cauchy formula, at the end changed $n$ ! to the Gamma function and then the definition of the fractional integral of non-integer order for $\alpha \in[n-1, n]$ can be written in form:

$D_{a}^{\alpha}(f)(t)=\frac{1}{\Gamma(n-\alpha)} \frac{d^{n}}{d t^{n}} \int_{a}^{t} \frac{f(x)}{(t-x)^{\alpha-n+1}} d x$.

Then, Caputo used the integrals to define fractional derivatives for $\alpha \in[n-1, n)$ in the form

$D_{a}^{\alpha}(f)(t)=\frac{1}{\Gamma(n-\alpha)} \int_{a}^{t} \frac{f^{(n)}(x)}{(t-x)^{\alpha-n+1}} d x$.

In another direction, Grunwald-Letnikov depended on repeated the time derivative $\alpha$ and then analyzing by using the Gamma function in the binomial coefficients [13-17]. But all the previous fractional derivatives were complicated, and they lost a lot of the main properties of ordinary derivatives like chain rule and product rule. Recently, it is appeared a new fractional derivative definition "the conformable fractional derivative" and that definition is perpendicular on the fundamentals definitions of the derivative for $0<\alpha \leq 1$ and $t>0$ :

$D^{\alpha} f(t)=\lim _{\varepsilon \rightarrow 0} \frac{f\left(t+\varepsilon t^{1-\alpha}\right)-f(t)}{\varepsilon}$,

and at zero the fractional derivative is knowing as $f^{\alpha}(0)=\lim _{t \rightarrow 0^{+}} f^{\alpha}(t)[18,19]$. When $\alpha=1$, this fractional derivative reduces to the ordinary derivative. The conformable fractional derivative has the following properties:

$$
\begin{array}{cc}
D^{\alpha} t^{p}=p t^{p-\alpha}, \quad p \in z, \quad D^{\alpha} c=0, \quad \forall f(t)=c, \\
D^{\alpha}(a f+b g)=a D^{\alpha} f+b D^{\alpha} g, \quad \forall a, b \in \mathbb{R}, \\
D^{\alpha}(f g)=f D^{\alpha} g+f D^{\alpha} g, \\
D^{\alpha} f(g)=\frac{d f}{d g} D^{\alpha} g, \quad D^{\alpha} f(t)=t^{1-\alpha} \frac{d f}{d g},
\end{array}
$$


where $f, g$ are two $\alpha$ - differentiable functions and $c$ is an arbitrary constant. The last equations are proved by Khalil et al. in [18]. The conformable fractional derivative of some functions

$$
\begin{aligned}
& D^{\alpha} e^{c t}=c t^{1-\alpha} e^{c t}, \quad D^{\alpha} \sin (c t)=c t^{1-\alpha} \cos (c t), \\
& D^{\alpha} \cos (c t)=-c t^{1-\alpha} \sin (c t), \\
& D^{\alpha} e^{c t^{\alpha}}=c \alpha e^{c t^{\alpha}}, \quad D^{\alpha} \sin \left(c t^{\alpha}\right)=c \alpha \cos \left(c t^{\alpha}\right), \\
& D^{\alpha} \cos \left(c t^{\alpha}\right)=-c \alpha \sin \left(c t^{\alpha}\right) .
\end{aligned}
$$

Karayer et al. [20] introduced the conformable fractional Nikiforov-Uvarov (NU) method for some prospect in quantum mechanics which gives accurate Eigen case solutions of Schrodinger equation (SE). Zhao et al. [21] discuss a new connotation of the delta conformable fractional derivative which has the identity factor on time scales. In, Ünal et al. [22] evidenced the power series solutions about given point in case of conformable fractional differential equations of linear sequential homogeneous of order $2 a$ and introduced the Hermite conformable fractional polynomials as well as the basic properties of these polynomials. Abu Hammad and Khalil [23] introduced the conformable fractional Fourier series. Cenesiz and Ali [24] establish the solution of conformable fractional heat equations for time and space by conformable Fourier transform. Jena and Chakraverty in [25] solved Navier-Stokes equations of fractional order by using a hybrid technique called homotopy perturbation Elzaki transform method. They merge Elzaki transform and homotopy perturbation method. This method was verified by using it on 3 different problems. It was shown that this proposed method is reliable, effective and easy to carry out various problems relating of science and engineering. Jena et al. [26] introduced the solution of a damped beam equation whose damping characteristics are well defined by the fractional derivative. They applied the homotopy analysis method for calculating the dynamic response and they compared the obtained results with the solutions achieved by Adomian decomposition method (ADM) to show the accuracy and efficiency of this method. Jena and Chakraverty [27] presented a new technique namely residual power series method to find the analytical solution of the Fractional Black-Scholes equation with an initial condition for European option pricing problem. Caputo sense is used to define the fractional derivative. This technique is based on expansion of the fractional power series. The method proved its effectiveness through the obtained solutions which compared with exact solutions solved by other techniques. Jena and Chakraverty [28] solved the time-fractional model of vibration equation of large membranes by using an iterative technique namely residual power series method (RPSM) and they used Caputo sense to define the fractional derivative. This method proved its efficacy and the results obtained are verified graphically.
In this leaf, the fractional derivative is applied to discuss the unsteady free convection flow for a slanted cavity contained a porous medium and checked the effects of the fractional parameter on properties of the heat transfer and fluid motion for various values of the inclination angle, Rayleigh numbers and the aspect ratio. Also, one of the aims of this research is to achieve the heat and fluid flow in irregular enclosures not only in case of ordinary derivatives but also in case of fractional derivatives. It is difficult to work with a slanted cavity so, by using the nonlinear axis transformations, the computational field is charted into an orthogonal shape as proposed in [29]. The finite difference method is used to find the numerical solution of the governing equations; this method is presented in $[30,31]$ with some modifications.

\section{Problem description and mathematical analysis}

The considered physical model is an enclosure containing porous medium fluid. Figure 1 shows that the wall length is $L$ and the tendency angle of the cavity walls is $\Phi . T_{h}$ refer to inclined left wall and $T_{c}$ refer to right walls, while the horizontal walls are thermally insulated. It assumed that Darcy's law is contract, the fluid is normal and the inertial effects and Bossiness fluid are ignored within the porous medium. Considering all these suppositions, the conservation equations in the fractional form are expressed as:

$D_{x}^{\alpha \alpha} \psi+D_{y}^{\alpha \alpha} \psi=-\frac{g K \beta}{v} D_{x}^{\alpha} \mathrm{T}$

$\sigma D_{t}^{\beta} T+u D_{x}^{\alpha} T+v D_{y}^{\alpha} T=\alpha\left(D_{x}^{\alpha \alpha} T+D_{y}^{\alpha \alpha} T\right)$

Equations (4) and (5) are a generalization of the following equations, see Nield and Bejan [1]:

$D_{y} u-D_{x} v=-\frac{g K \beta}{v} D_{x} \mathrm{~T}$.

$\sigma D_{t} T+u D_{x} T+v D_{y} T=\alpha\left(D_{x x} T+D_{y y} T\right)$.

where $u=\frac{\partial \psi}{\partial y}, v=-\frac{\partial \psi}{\partial x}$ are the velocity components and $D^{\alpha}$ is the fractional differential operator.

Introducing the following grid transformations:

$X=x-y \tan \Phi, Y=y$

$D_{x}=D_{X}, D_{y}=D_{Y}-\tan \Phi D_{X}$

Substituting Eqs. (8) and (9) into Eqs. (4) and (5), the following system is obtained:

$D_{X}^{\alpha \alpha} \psi-2 \sin \Phi \cos \Phi D_{X}^{\alpha}\left(D_{Y}^{\alpha} \psi\right)+\cos ^{2} \Phi D_{Y}^{\alpha \alpha} \psi=-\frac{g K \beta}{v} D_{X}^{\alpha} T \cos ^{2} \Phi$. 


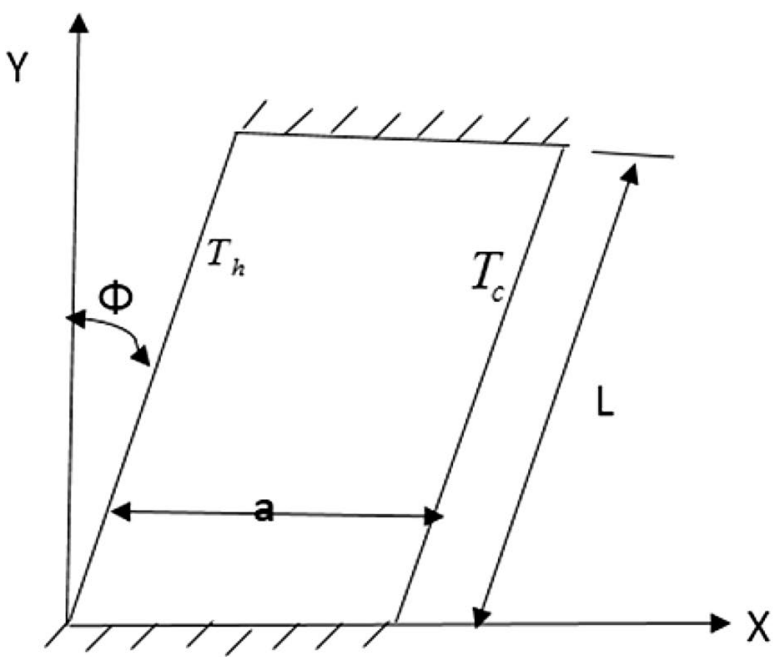

(a)

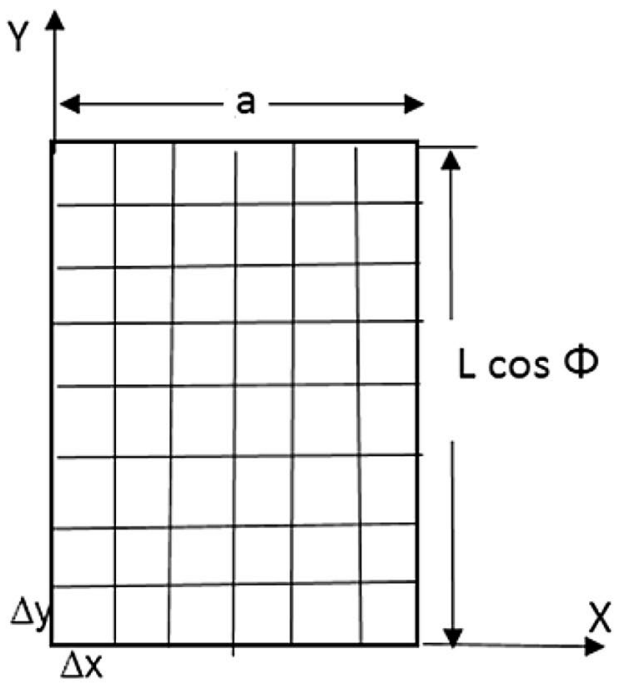

(b)

Fig. 1 a Physical model and $\mathbf{b}$ transformed computational domain

$\sigma D_{t}^{\beta}+D_{y}^{\alpha} \psi D_{x}^{\alpha} T-D_{x}^{\alpha} \psi D_{y}^{\alpha} T=\frac{\alpha}{\cos ^{2} \Phi}\left[D_{x}^{\alpha \alpha} T-2 \sin \Phi \cos \Phi D_{x}^{\alpha}\left(D_{y}^{\alpha} T\right)+\cos ^{2} \Phi D_{y}^{\alpha \alpha} T\right]$.

Using the following dimensionless parameter:

$\eta=\mathrm{Y} /(\mathrm{L} \cos \Phi), \quad \tau=t(\bar{\alpha} / \sigma a L \cos \Phi), \quad \zeta=X / a$,

$\Psi=\psi / \bar{\alpha}, \quad \theta=\left(\mathrm{T}-\mathrm{T}_{r}\right) / \Delta T$

where $T_{r}=\left(T_{h}+T_{c}\right) / 2$ and $\Delta \mathrm{T}=\mathrm{T}_{h}-T_{c}$ with $\mathrm{T}_{h}>T_{c}$.

Substituting Eq. (12) into Eqs. (10), (11), the following system is obtained:

$D_{\zeta}^{\alpha \alpha} \Psi-2 \frac{\sin \Phi}{A} D_{\zeta}^{\alpha}\left(D_{\eta}^{\alpha} \Psi\right)+\frac{1}{A^{2}} D_{\eta}^{\alpha \alpha} \Psi=-R a \cos ^{2} \Phi D_{\zeta}^{\alpha} \theta$.

$$
\begin{aligned}
& \frac{A}{\cos \Phi}\left(D_{\zeta}^{\alpha \alpha} \theta-\frac{2 \sin \Phi}{A} D_{\zeta}^{\alpha}\left(D_{\eta}^{\alpha} \theta\right)+\frac{1}{A^{2}} D_{\eta}^{\alpha \alpha} \theta\right) \\
& \quad=D_{\eta}^{\alpha} \Psi D_{\zeta}^{\alpha} \theta+D_{\tau}^{\beta} \theta-D_{\zeta}^{\alpha} \Psi D_{\eta}^{\alpha} \theta .
\end{aligned}
$$

where $A$ is the aspect ratio of the cavity and $R a$ is the Rayleigh number:

$A=a / L, \quad \mathrm{Ra}=\frac{a \Delta T \bar{\beta} g k}{v \bar{\alpha}}$

The subjected boundary conditions are given by:

$$
\begin{array}{ll}
\Psi=0, \quad \theta=\frac{1}{2}, \quad \text { on } \zeta=0 \\
\Psi=0, \quad \theta=-\frac{1}{2} \quad \text { on } \zeta=1 \\
\Psi=0, \quad \frac{\partial \theta}{\partial \eta}-A \sin \Phi \frac{\partial \theta}{\partial \zeta}=0 \quad \text { on } \eta=0,1
\end{array}
$$

The local Nusselt number takes the form:

$N u(\zeta, \eta)=\frac{a q_{w}}{\Delta T k}$

Since, $q_{w}$ is the heat transfer rate, it can be evaluated at the slanted walls as:

$q_{w}=-k n . \nabla T$,

In Eq. (18), $k$ is the porous medium thermal conductivity and $n$ is the unit vector normal to the slanted walls.

$n=\{-\cos \Phi i, \sin \Phi j\}$.

Substituting Eqs. (16) and (17) in Eq. (15), the local Nusselt number being in the form:

$N u(\zeta, \eta)=\frac{-1}{\cos \Phi}\left(\frac{\sin \Phi}{A} \frac{\partial \theta}{\partial \eta}-\frac{\partial \theta}{\partial \zeta}\right)_{\zeta=0,1}$

Also, the average Nusselt number is given by:

$\overline{N u}(\zeta)=\int_{0}^{1} N u(\zeta, \eta) d \eta$

\section{Numerical method and validation}

In this part, the finite differences method is applied to find numerically the solution of the fractional partial differential Eqs. (12) and (13) subjected to the boundary conditions (15). The fractional derivatives are approximated using the 
definition in Eq. (3), then the second differences approaches are used to implement the first and second derivatives. The resulting algebraic system is solved using SUR method with successive parameter $\varepsilon=0.7$. The grid $101 \times 101$ is chosen for all computations after conducting a grid dependency study. The convergence criteria of $10^{-6}$ are applied to terminate the loops and many validation tests are reported.

\subsection{The finite difference method in the fractional derivative}

Since Guranwald letnikov derivatives with order $a>0$ defined as follow:

$D_{a, t}^{\alpha} f(t)=\lim _{h \rightarrow 0, N h \rightarrow t-a} h^{-\alpha} \sum_{j=0}^{N}(-1)^{j}\left(\begin{array}{c}\alpha \\ j\end{array}\right) f(t-j h)$

The Riemann-Liouville derivatives for $a>0$ in the form

$\mathrm{D}_{\mathrm{a}}^{\alpha} \mathrm{f}(\mathrm{t})=\frac{1}{\Gamma(n-\alpha)} \frac{d^{n}}{d t^{n}} \int_{a}^{t}(t-s)^{n-\alpha-1} f(s) d s$

where $n$ is an integer number and $n-1 \leq \alpha<n$.

The caputo definition for $a>0$

$\mathrm{CD}_{\mathrm{a}}^{\alpha} \mathrm{f}(\mathrm{t})=\frac{1}{\Gamma(n-\alpha)} \int_{a}^{t}(t-s)^{n-\alpha-1} f^{(n)}(s) d s$

The relation between Riemann and Caputo given in the following

$D_{a, t}^{\alpha} f(t)=c D_{a, t}^{\alpha} f(t)+\sum_{k=0}^{n-1} \frac{f^{(k)}(a)(t-a)^{k-a}}{\Gamma(1-\alpha)}$

In Eq. (22) let $\omega_{j}^{\alpha}=(-1)^{j}\left(\begin{array}{c}\alpha \\ j\end{array}\right)$.

Then

$D_{t_{0}, t}^{\alpha} u(t)=\lim _{h \rightarrow 0, N h \rightarrow t-t 0} \Delta T^{-\alpha} \sum_{j=0}^{N} \omega_{j}^{\alpha} u\left(t_{k-j}\right)$

where $u(t)$ is defined on $\left[t_{0}, t\right], \Delta t=\frac{\left(t_{0}-t\right)}{n_{t}}$ (uniform time step), $n_{t}$ is a positive integer $t_{k}$ is the temporal grid points, $t_{k}=t_{0}+k \Delta t, \mathrm{k}=0, \ldots, n_{t}$.

Then the Riemann-Liouville derivative can be approximated

$D_{t_{0}, t}^{\alpha} u\left(t_{k}\right) \approx D_{t_{0}, t}^{\alpha} \check{u}\left(t_{k}\right)=\Delta t^{-\alpha} \sum_{j=0}^{k} \omega_{j}^{\alpha} u\left(t_{k-j}\right)$
Equation (27) is called slandered formula, for $1<\alpha<2$ the formula is more stable

$D_{t_{0}, t}^{\alpha} u\left(t_{k}\right) \approx D_{t_{0}, t}^{\check{\alpha}} u\left(t_{k}\right)=\Delta t^{-\alpha} \sum_{j=0}^{k} \omega_{j}^{\alpha} u\left(t_{k-j+1}\right)$

The relation (28) has one order convergence which the numerical scheme is more stable $\left\{\omega_{j}^{\alpha}\right\}_{j=0}^{k}$ is the first $k+1$ coefficient of the Taylor series.

The active way to approximate the Riemann-Liouville derivative of order $\alpha(0<\alpha<1)$ by using the Eq. (25):

$D_{a, t}^{\alpha} f(t)=\mathrm{CD}_{a, t}^{\alpha} f(t)+\sum_{k=0}^{n-1} \frac{f^{(k)}(a)(t-a)^{k-a}}{\Gamma(1-\alpha)}$

The $L_{1}$ scheme obtained in form:

$$
\begin{aligned}
D_{t_{0}, t}^{\alpha} u\left(t_{k+1}\right)= & \frac{u\left(t_{0}\right)\left(t_{k+1}-t_{0}\right)}{\Gamma(1-\alpha)} \\
& +\frac{1}{\Gamma(1-\alpha)} \sum_{j=0}^{k} \int_{t_{j}}^{t_{j+1}}\left(t_{k+1}-s\right)^{-\alpha} u^{\prime}(s) d s
\end{aligned}
$$

where $\int_{t_{j}}^{t_{j+1}}\left(t_{k+1}-s\right)^{-\alpha} u^{\prime}(s) d s$ is approximated by $\int_{t_{j}}^{t_{j+1}} \frac{u\left(t_{j+1}\right)-\left(t_{j}\right)}{\Delta t}\left(t_{k+1}-s\right)^{-\alpha} d s$, then $L_{1}$ scheme is:

$D_{t_{0}, t}^{\alpha} \check{u}\left(t_{k+1}\right)=\frac{u\left(t_{0}\right)\left(t_{k+1}-t_{0}\right)}{\Gamma(1-\alpha)}+\frac{\Delta t^{-\alpha}}{\Gamma(2-\alpha)} \sum_{j=0}^{k} b_{j, k}\left[u\left(t_{j+1}\right)-u\left(t_{j}\right)\right]$

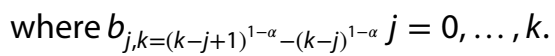

In the similar manner the $L_{2}$ scheme in case of $\alpha(1<\alpha<2)$ is given by:

$$
\begin{aligned}
D_{t_{0}, t}^{\alpha} u\left(t_{k+1}\right)= & \frac{u\left(t_{0}\right)\left(t_{k+1}-t_{0}\right)^{-\alpha}}{\Gamma(1-\alpha)}+\frac{u^{\prime}\left(t_{0}\right)\left(t_{k+1}-t_{0}\right)^{1-\alpha}}{\Gamma(2-\alpha)} \\
& +\frac{1}{\Gamma(2-\alpha)} \sum_{j=0}^{k} \int_{t_{j}}^{t_{j+1}} s^{1-\alpha} u^{\prime \prime}\left(t_{k+1}-s\right) d s
\end{aligned}
$$

$u^{\prime \prime}\left(t_{k+1}-s\right) \approx \frac{u\left(t_{k-j-1}\right)-2 u\left(t_{k-j}\right)+u\left(t_{k+1-j}\right)}{\Delta t^{2}}$

By using (32) in (33) 


$$
\begin{aligned}
D_{t_{0}, t}^{\alpha} \check{u}\left(t_{k+1}\right)= & \frac{u\left(t_{0}\right)\left(t_{k+1}-t_{0}\right)^{-\alpha}}{\Gamma(1-\alpha)}+\frac{u^{\prime}\left(t_{0}\right)\left(t_{k+1}-t_{0}\right)^{1-\alpha}}{\Gamma(2-\alpha)} \\
& +\frac{\Delta t^{-\alpha}}{\Gamma(3-\alpha)} \sum_{j=0}^{k} b_{j}\left[u\left(t_{k-j-1}\right)-2 u\left(t_{k-j}\right)+u\left(t_{k+1-j}\right)\right] \\
= & \frac{u\left(t_{0}\right)\left(t_{k+1}-t_{0}\right)^{-\alpha}}{\Gamma(1-\alpha)}+\frac{u^{\prime}\left(t_{0}\right)\left(t_{k+1}-t_{0}\right)^{1-\alpha}}{\Gamma(2-\alpha)} \\
& +\sum_{j=-1}^{k+1} W_{j} u\left(t_{k+1-j}\right)
\end{aligned}
$$

where $b_{j}=(j-1)^{2-\alpha}-j^{2-\alpha}$ and $\left\{W_{j}\right\}_{j=-1}^{k+1}$ can be expressed by $\left\{b_{j}\right\}_{j=0}^{k}$ by defining $u^{\prime \prime}\left(t_{k+1}-s\right)$ in subinterval $\left[t_{j,} t_{k j+1}\right]$ in the form:

$u^{\prime \prime}\left(t_{k+1}-s\right) \approx \frac{u\left(t_{k-j-1}\right)-u\left(t_{k-j}\right)+u\left(t_{k+2-j}\right)-u\left(t_{k+1-j}\right)}{2 \Delta t^{2}}$

A new scheme is obtained, namely, $L_{2} C$ in the form:

$$
\begin{aligned}
D_{t_{0}, t}^{\alpha} \check{u}\left(t_{k+1}\right)= & \frac{u\left(t_{0}\right)\left(t_{k+1}-t_{0}\right)^{-\alpha}}{\Gamma(1-\alpha)}+\frac{u^{\prime}\left(t_{0}\right)\left(t_{k+1}-t_{0}\right)^{1-\alpha}}{\Gamma(2-\alpha)} \\
& +\frac{\Delta t^{-\alpha}}{2 \Gamma(3-\alpha)}\left[u\left(t_{k-j-1}\right)-u\left(t_{k-j}\right)+u\left(t_{k+2-j}\right)-u\left(t_{k+1-j}\right)\right] \\
= & \frac{u\left(t_{0}\right)\left(t_{k+1}-t_{0}\right)^{-\alpha}}{\Gamma(1-\alpha)}+\frac{u^{\prime}\left(t_{0}\right)\left(t_{k+1}-t_{0}\right)^{1-\alpha}}{\Gamma(2-\alpha)} \\
& +\sum_{j=-1}^{k+2} W_{j} u\left(t_{k+1-j}\right)
\end{aligned}
$$

When $\alpha=1$, the scheme $L_{1}$ in Eq. (28) is reduced to the back forward difference, $L_{2}$ in Eq. (31) is reduced to the forward difference method and $L_{2} C$ in Eq. (32) is reduced to the centered difference method, see [32-34]:

\subsection{Validation}

Table 1 shows comparisons of the average Nusselt for varies values of $R a(R a=100,1000,100,00)$ with many

Table 1 Comparisons of the average Nusselt number at $A=1$ and $\Phi=0$

\begin{tabular}{llll}
\hline Authors & \multicolumn{2}{l}{$\mathrm{Ra}$} & \\
\cline { 2 - 4 } & 100 & 1000 & 10,000 \\
\hline Baytas and Pop [3] & 3.6 & 14.06 & 48.33 \\
Walker and Homsy [35] & 3.97 & 12.96 & 51 \\
Gross et al. [37] & 3.141 & 13.448 & 42.583 \\
Bejan [36] & 4.2 & 15.8 & 50.8 \\
Manole and Lage [31] & 3.118 & 13.637 & 48.33 \\
Present result at ordinary & 2.6779 & 15.07595 & 57.15578 \\
$\quad$ case ( $a=1$ ) & & & \\
Present result at $(\mathrm{a}=0.9)$ & 3.897 & 21.31477 & 84.26131 \\
\hline
\end{tabular}

published results (Baytas and Pop [3], Walker and Homsy [35], Bejan [36], Gross et al. [37] and Manole and Lage [31]) in case of the ordinary derivatives $a=1$. It is noted that there are good harmony between the results. Also, it is worth mentioned that a home FORTRAN code is used and, approximately, 5 min CPU time is the time of each computation.

\section{Results and discussion}

The obtained results are shown in terms of contours of the isotherms and streamlines. Also, profiles of the Nusselt and average Nusselt number at the hot wall. In this simulation, orders of the fractional derivatives take the range from 0.7 to 1 , the walls inclination angle is varied from 0 to $45^{\circ}$, values of Rayleigh number are between $10^{2}$ and $10^{4}$ and the aspect ratio is fixed at $A=1$.

Table 2 presents results of the average Nusselt for some values of the fractional derivatives orders $a$ and $\beta$ at $R a=100,1000,100,00$. It is observed that the decrease in time fractional derivatives order $\beta$ gives a small reduction in values of $\mathrm{Nu}$. This behavior is observed only in case of $\mathrm{Ra}=100$ but in other cases $(R a=1000,100,00)$ the average Nusselt number is insensitive with variations of $\beta$. In addition, the variations of $a$ are more dominant on behaviors of $N u$ than $\beta$. A very good enhancement in values of $N u$ is noted as $a$ decreases from 1 to 0.9 .

Figures 2, 3 and 4 display contours of the isotherms and streamlines for various values the fractional derivatives orders $\alpha$ and $\beta$ in cases of $\Phi=0, \Phi=30^{\circ}$ and $\Phi=45^{\circ}$ respectively. In all these figures, the Rayleigh number is fixed at $R a=1000$. The results revealed that, in general, the streamlines show a large clockwise circular cell inside the enclosure with limited increment between the minimum value of $\Psi_{\text {min }}$ at the boundaries and maximum value $\Psi_{\text {max }}$. Also, the isotherms are distributed with equal distances from the greatest value of temperature of the hot wall to the lowest value of temperature of the cold wall. In addition, the decrease in the derivatives orders $a$

Table 2 Results of average Nusselt number for various values of $\alpha, \beta$ and $R a$

\begin{tabular}{llll}
\hline$\alpha$ and $\beta$ & \multicolumn{3}{l}{} \\
\cline { 2 - 4 } & $\mathrm{Ra}=100$ & $\mathrm{Ra}=1000$ & $\mathrm{Ra}=10,000$ \\
\hline$\alpha=1, \beta=1$ & 2.677966 & 15.07595 & 57.15578 \\
$\alpha=1, \beta=0.9$ & 2.692308 & 15.07595 & 57.15578 \\
$\alpha=1, \beta=0.8$ & 2.692308 & 15.07595 & 57.15578 \\
$\alpha=0.9, \beta=1$ & 3.897436 & 21.34177 & 84.26131 \\
$\alpha=0.8, \beta=1$ & 5.384615 & 29.18987 & 121.4573 \\
$\alpha=0.8, \beta=0.8$ & 5.34615 & 29.18987 & 121.4573
\end{tabular}



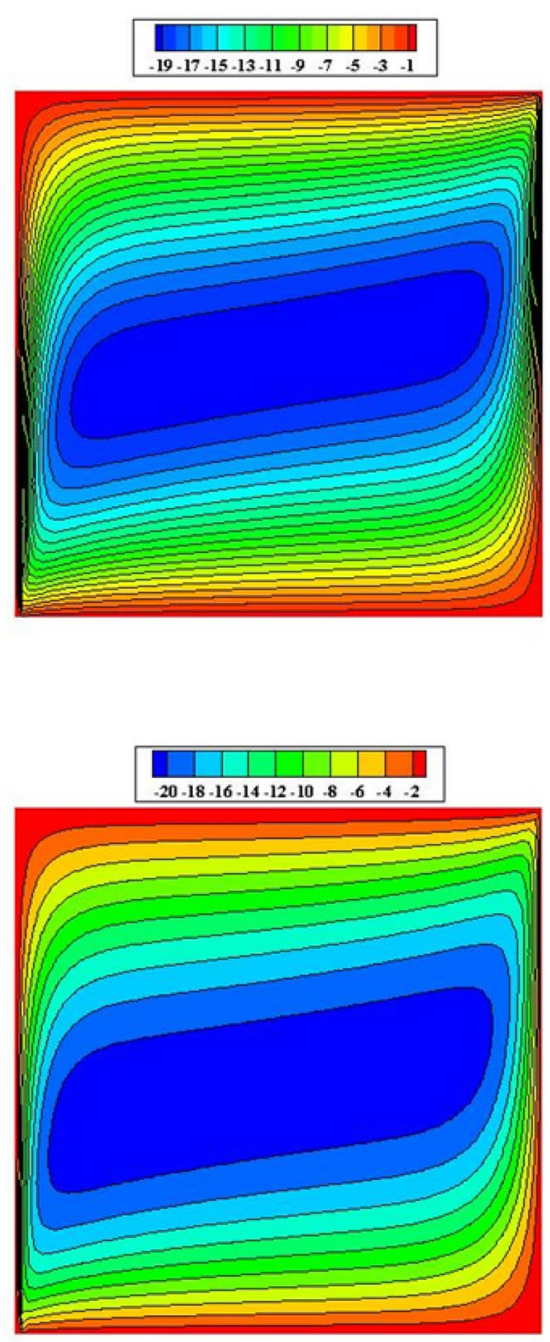

(b) $\alpha=0.9, \beta=1$

(a) $\alpha=1, \beta=1$
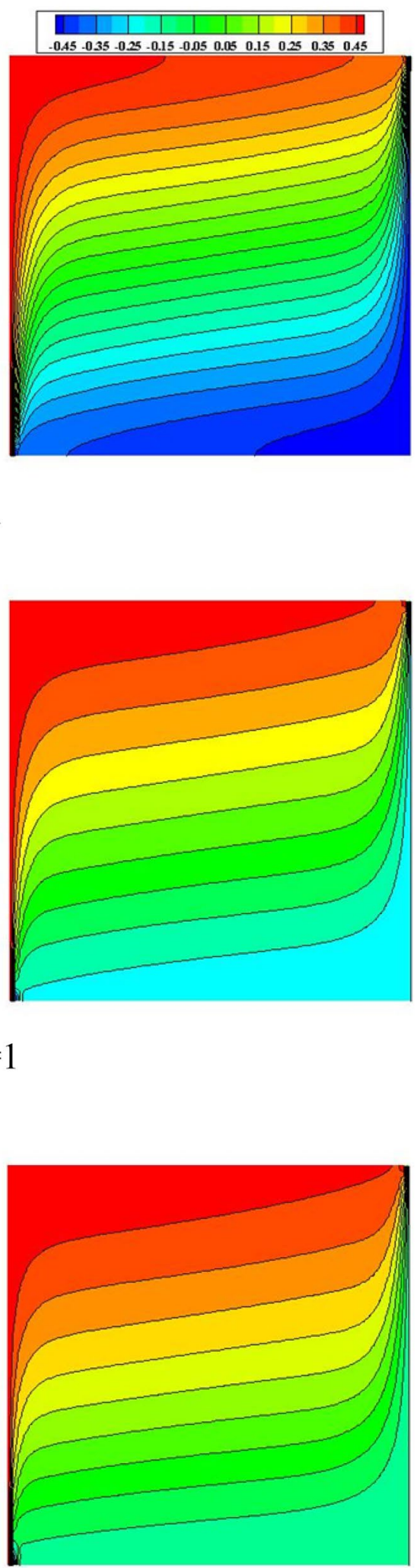

(c) $\alpha=0.8, \beta=1$

Fig. 2 Streamlines and isotherms for various values of $a$ and $\beta$ at $A=1, \Phi=0^{\circ}, R a=10^{3}$ 

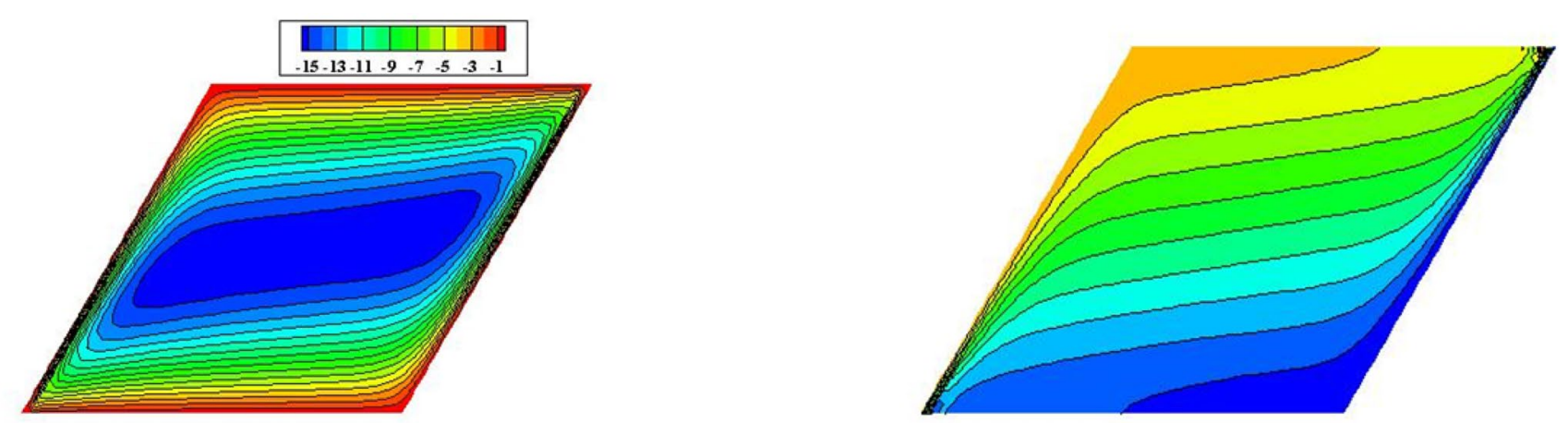

(a) $\alpha=1, \beta=1$
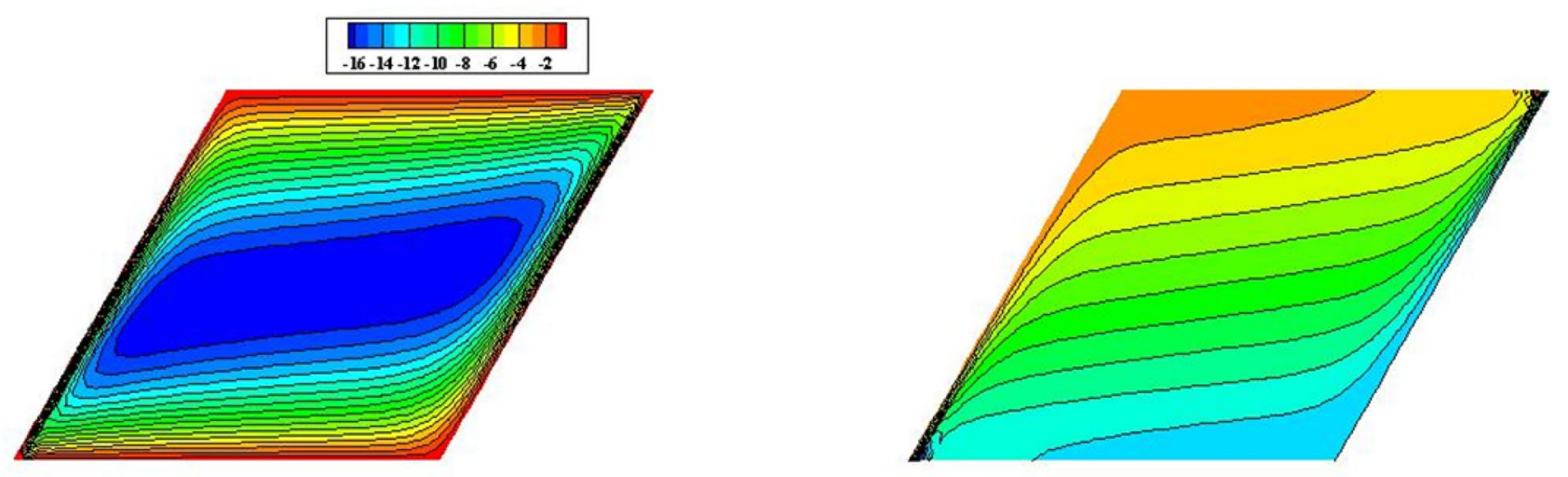

(b) $\alpha=0.9, \beta=0.9$
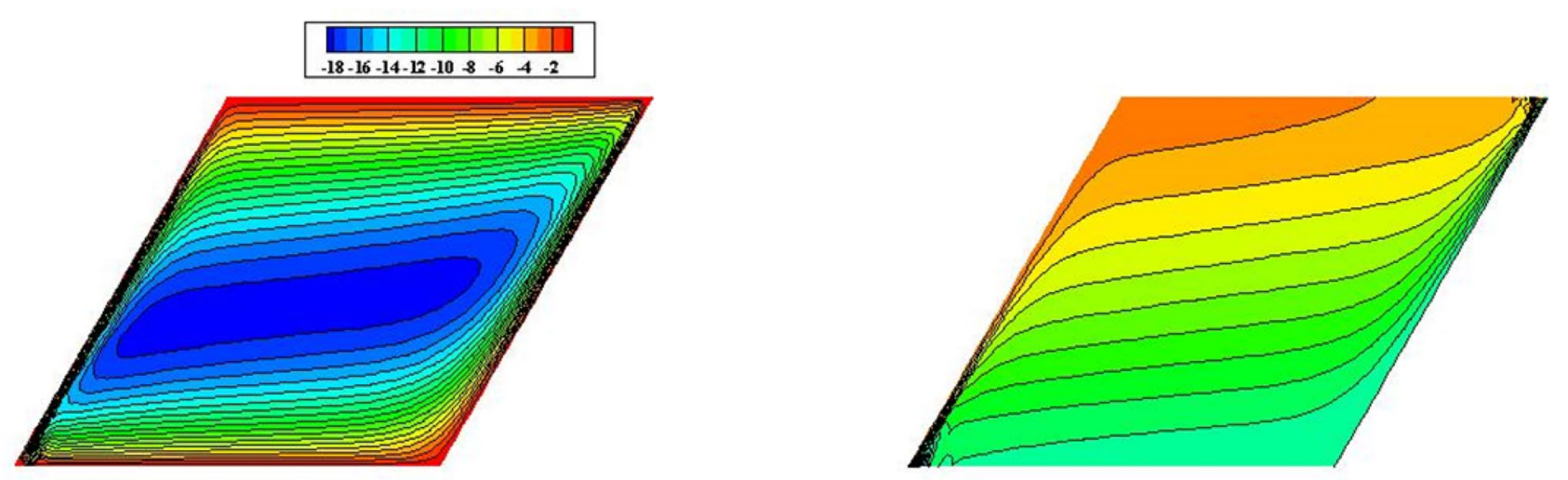

(c) $\alpha=0.8, \beta=0.8$

Fig. 3 Streamlines and isotherms for various values of $a$ and $\beta$ at $A=1, \Phi=30^{\circ}, \operatorname{Ra}=10^{3}$

and $\beta$ accelerates the fluid flow and enhances the thermal boundary layer near the left wall. On the contrary, the increase in the inclination angle $\Phi$ acts as a retarding force to the fluid movement. It reduces the maximum values the stream function as $\Phi$ decreases. Also, the thermal boundary layer near the heated wall is diminished with the 

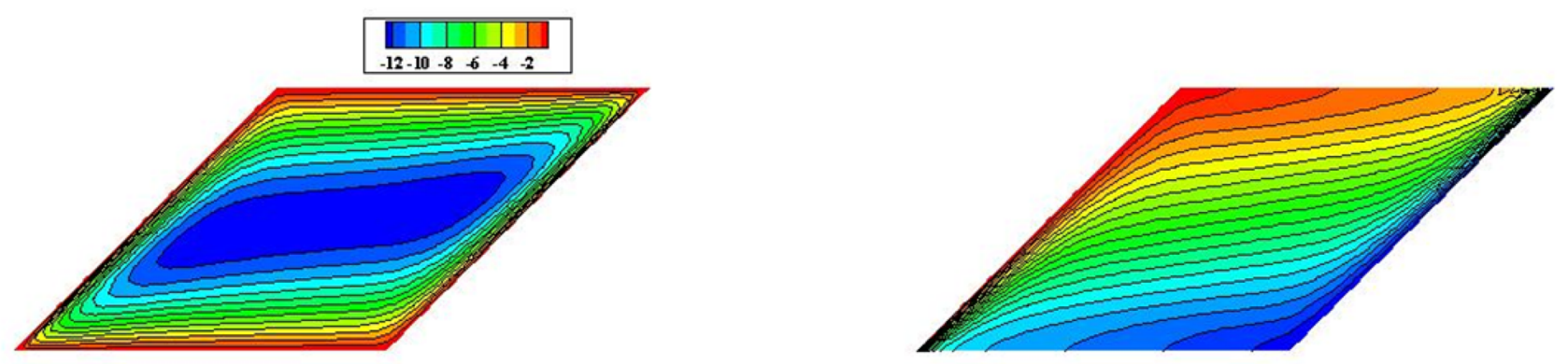

(a) $\alpha=1, \beta=1$
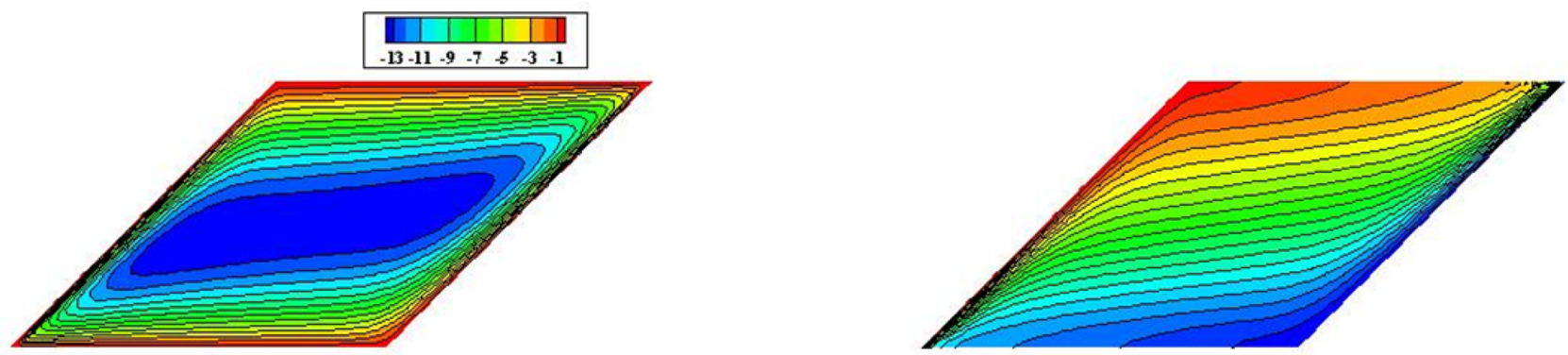

(b) $\alpha=0.9, \beta=0.9$
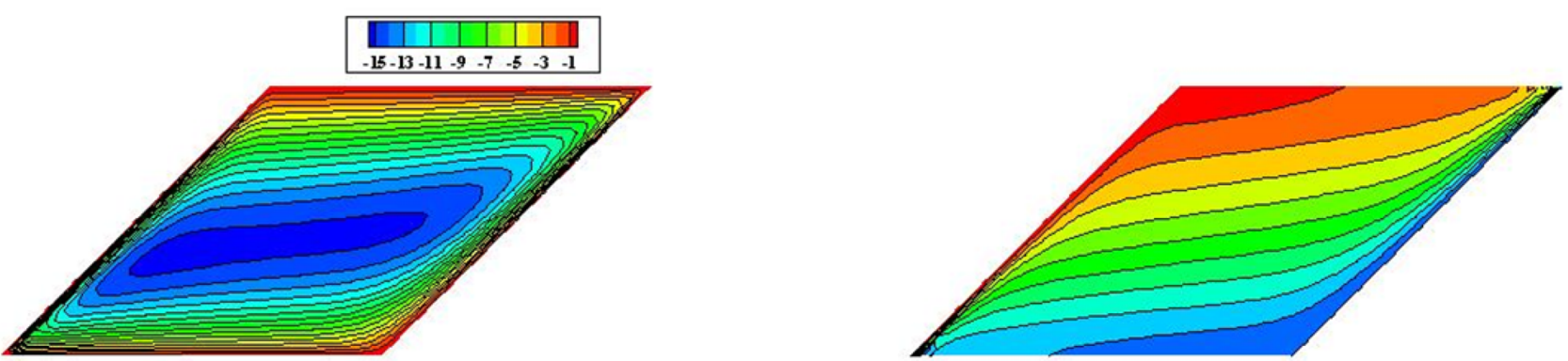

(c) $\alpha=0.8, \beta=0.8$

Fig. 4 Streamlines and isotherms for various values of $a$ and $\beta$ at $A=1, \Phi=45^{\circ}$, $R a=10^{3}$

growing of $\Phi$. Physical, when $\Phi$ is increased the geometry of the enclosure become more complex and consequently the natural convection is reduced.

In Fig. 5a-c, profiles of the Nusselt numbers at the hot wall for variations of the fractional derivatives orders $a$ and $\beta$ are plotted. Also, these figures corresponds the cases of square enclosure $(\Phi=0)$ and slanted enclosures $\left(\Phi=30^{\circ}\right.$,
$\Phi=45^{\circ}$ ) respectively. It is found that the Nusselt number decreases monotonically as $a$ and $\beta$ decreases. Like effect of $\alpha$ and $\beta$, the increases in $\Phi$ results in a reduction in the local Nusselt due to the decrease in the thermal boundary layer mentioned later. Moreover, as it be observed from Fig. 6 which show the average Nusselt number for 

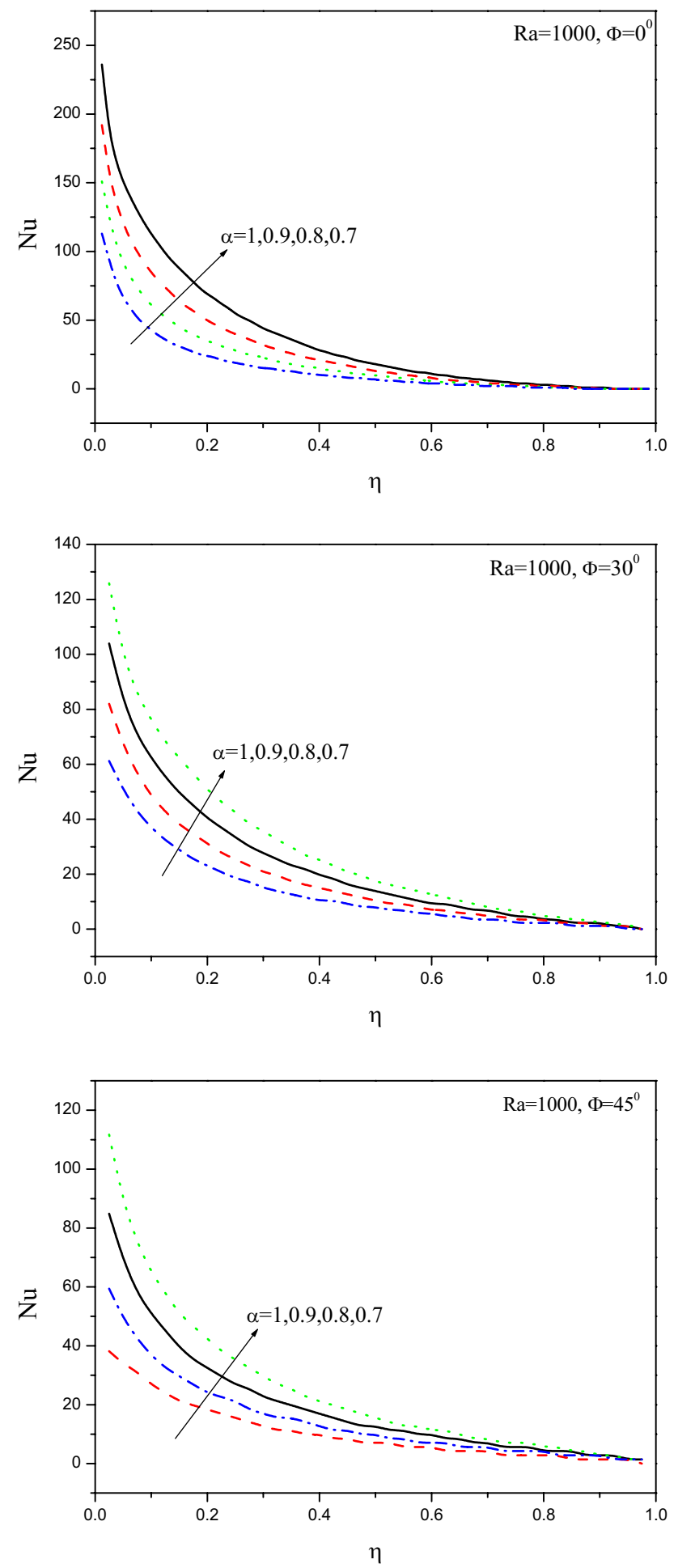

Fig. 5 a Nusselt number at the hot wall for various values of $a$ at $\Phi=0^{\circ}, \mathrm{Ra}=10^{3}$. b Nusselt number at the heated wall for various values of a at $\Phi=30^{\circ}, R a=10^{3}$. c Nusselt number at the heated wall for various values of a at $\Phi=45^{\circ}, \mathrm{Ra}=10^{3}$

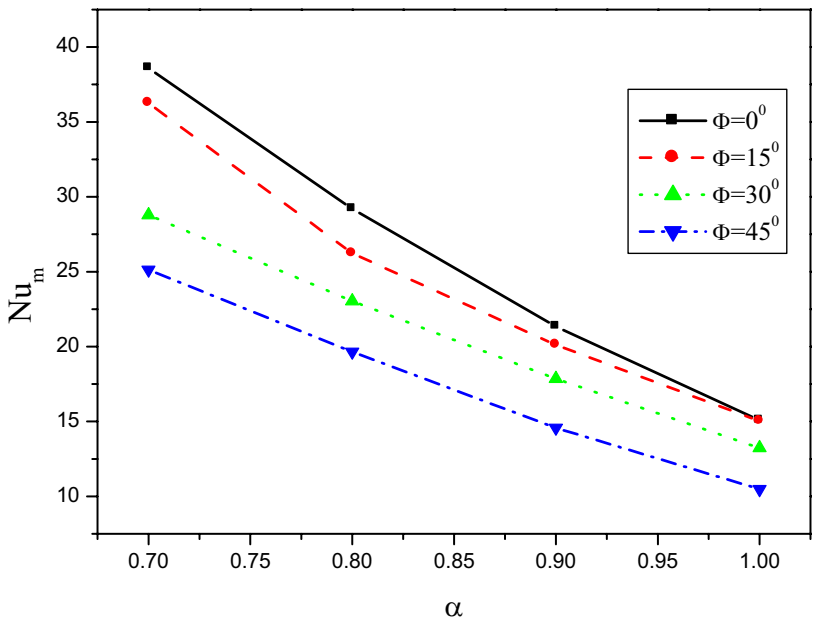

Fig. 6 Average Nusselt number at the hot wall for various values of $a$ and $\Phi$ at $\operatorname{Ra}=10^{3}$

variations of $a, \beta$ and $\Phi$, a clear reduction in profiles of $N u$ is noted as either $a$ and $\beta$ or $\Phi$ decrease.

With the help of Figs. 7, 8, 9 and 10, effects of variation the fractional derivatives $a$ and $\beta$ on the contours of the isotherms and streamlines at $R a=10^{4}$. In these figures, values of the walls inclination angle $\Phi$ are taken to be equal $0^{\circ}, 15^{\circ}, 30^{\circ}$ and $45^{\circ}$, respectively. By comparing case of $R a=10^{3}$ with the current case, one can observe that great enhancements in rate of the fluid flow are found in case of $R a=10^{4}$ due to the increase in the buoyancy force. In addition, like case of $R a=10^{3}$, the decrease in $a$ and $\beta$ enhances the minimum values the stream function indicating a good natural convective transport. But as the inclination angle increases, negative effects on the streamlines and temperature distributions are noted regardless values of the Ra, $a$ and $\beta$.

Figures 11 and 12 show rate of transfer of heat represented by profiles of the local and average Nusselt numbers for some values of the fractional derivatives orders $a$ and $\beta$ and, also, for variations of the inclination angle in case of the high Rayleigh number $\left(R a=10^{4}\right)$. It is clear that a significant reduction in both of the local and average Nusselt number is found as the fractional derivatives orders $\alpha$ and $\beta$ decreases. Also, similar behaviors are noted as the inclination angle increases. Physically, these behaviors are due to the reduction in the thermal boundary layer near the left wall that is obtained as $a$ and $\beta$ decreases or $\Phi$ increases. 

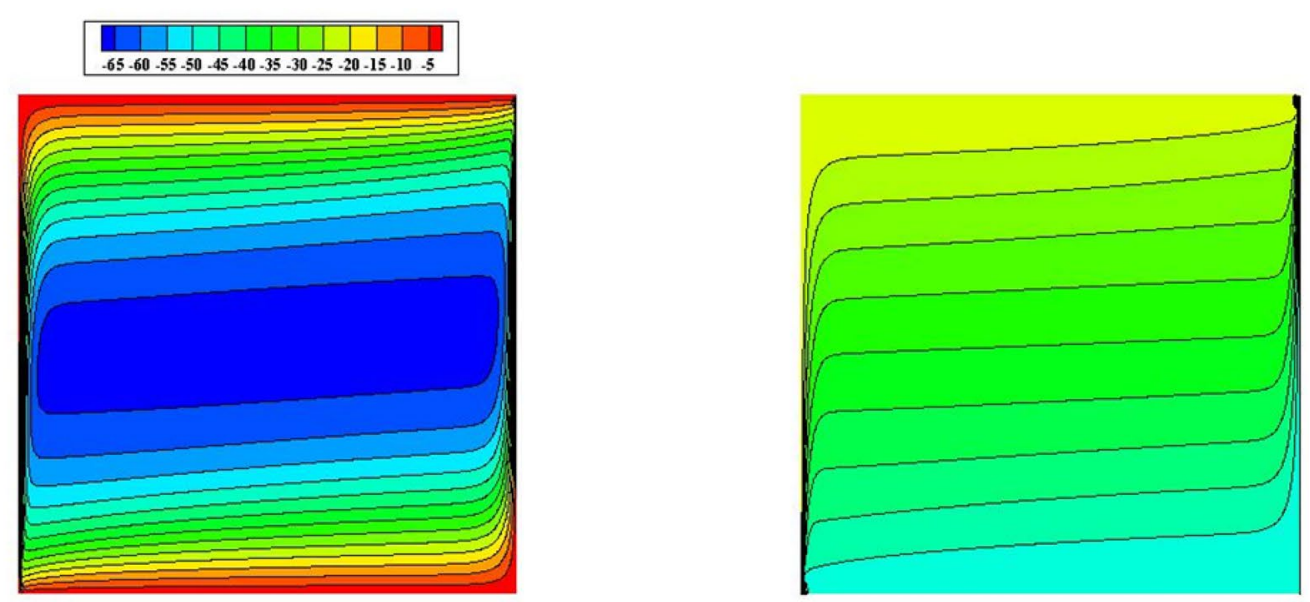

(a) $\alpha=1, \beta=1$
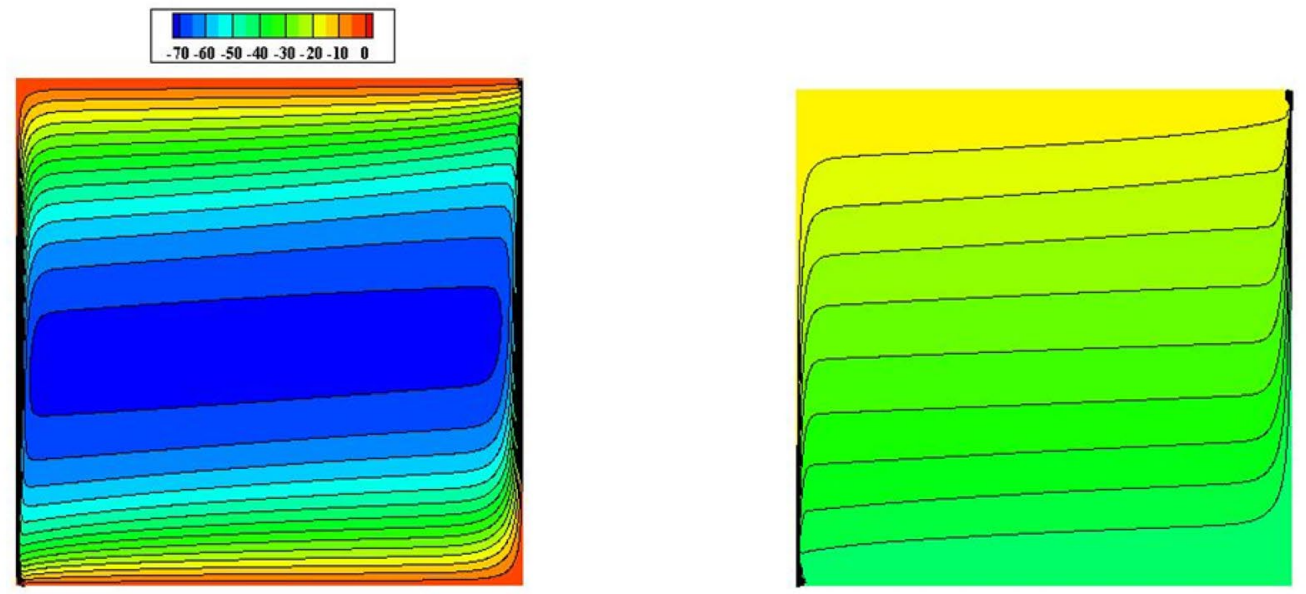

(b) $\alpha=0.9, \beta=1$
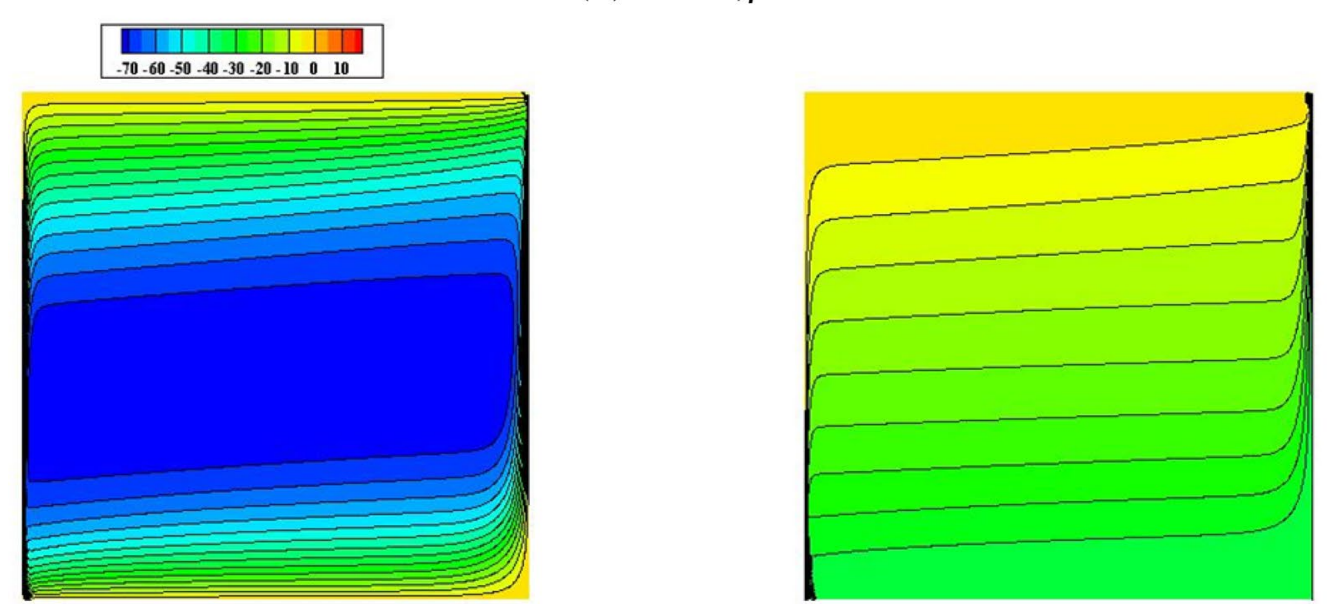

(c) $\alpha=0.8, \beta=1$

Fig. 7 Streamlines and isotherms for different values of $\alpha$ and $\beta$ at $A=1, \Phi=0^{\circ}, \operatorname{Ra}=10^{4}$ 

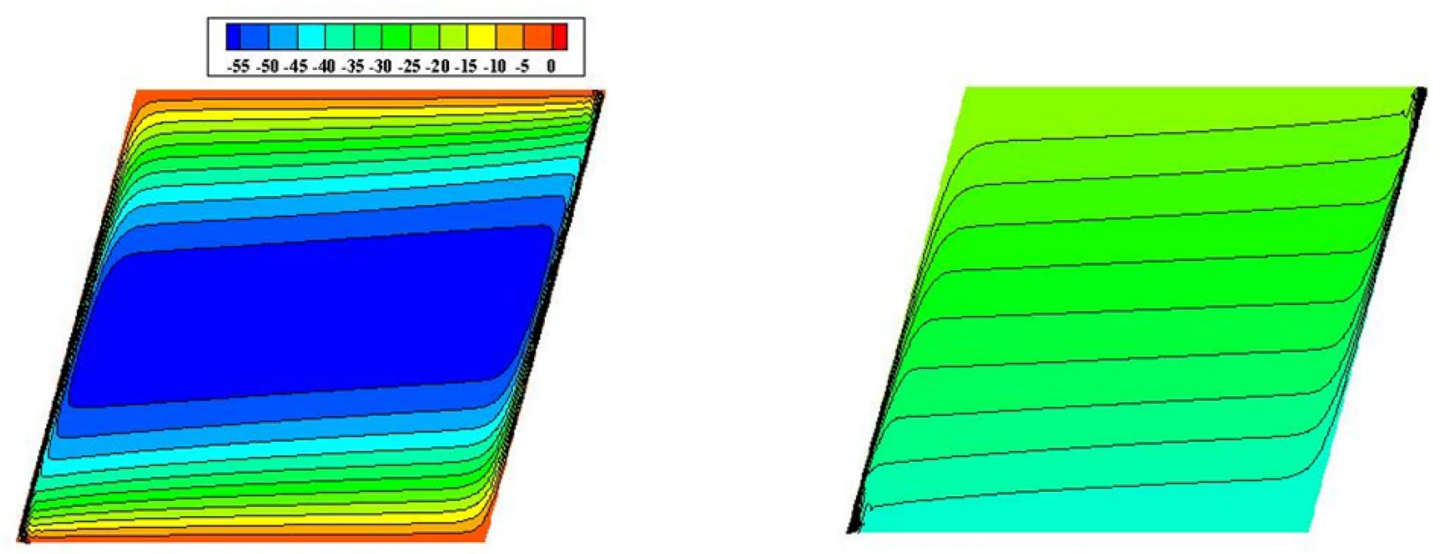

(a) $\alpha=1, \beta=1$
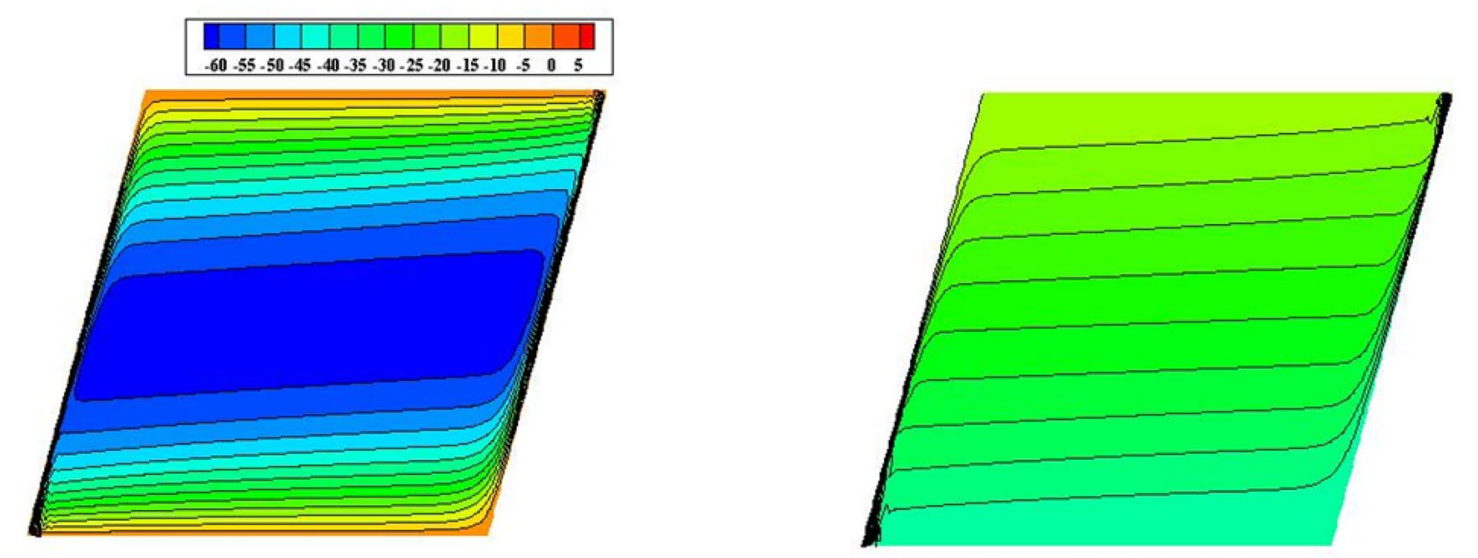

(b) $\alpha=0.9, \beta=0.9$
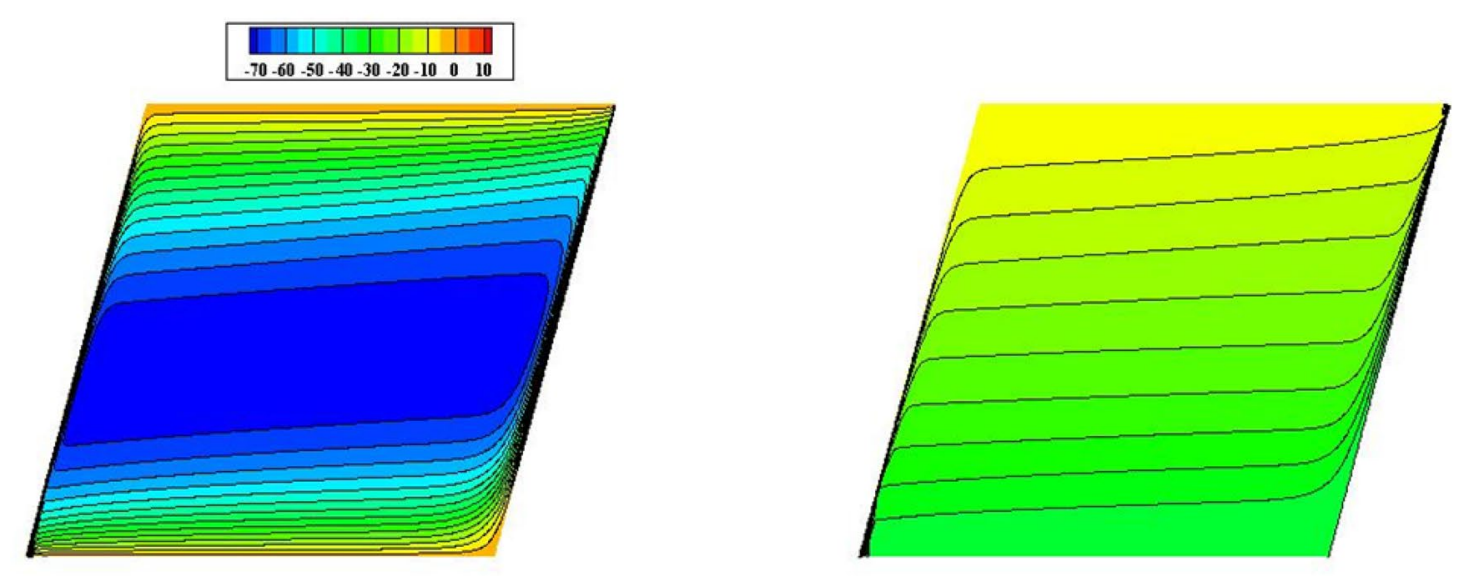

(c) $\alpha=0.8, \beta=0.8$

Fig. 8 Streamlines and isotherms for various values of $a$ and $\beta A=1, \Phi=15^{\circ}, R a=10^{4}$ 

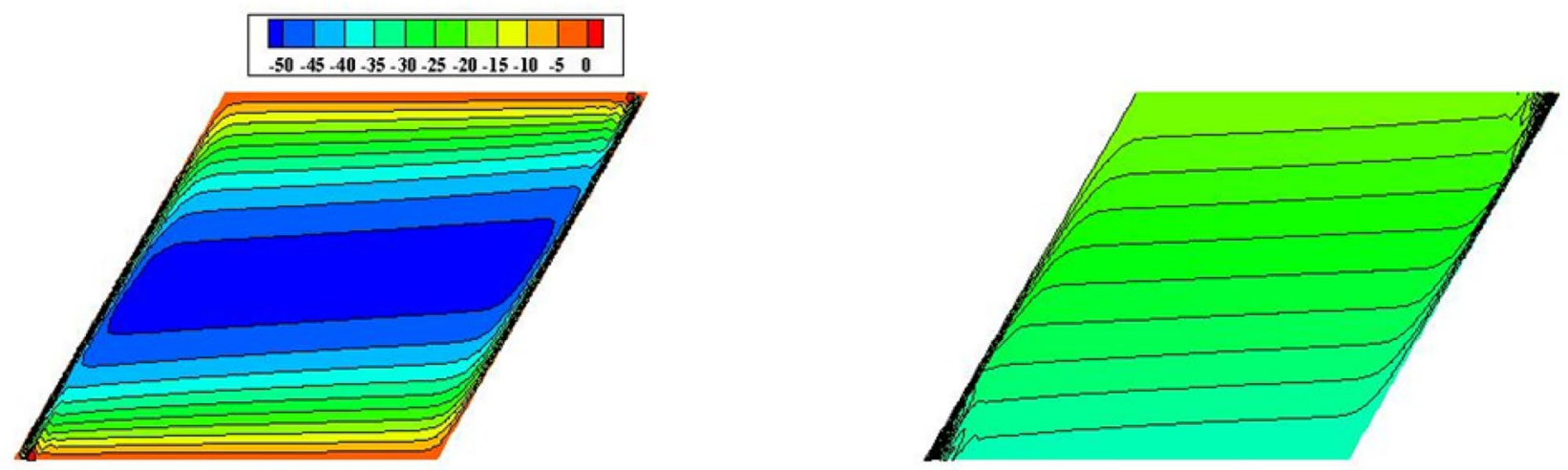

(a) $\alpha=1, \beta=1$
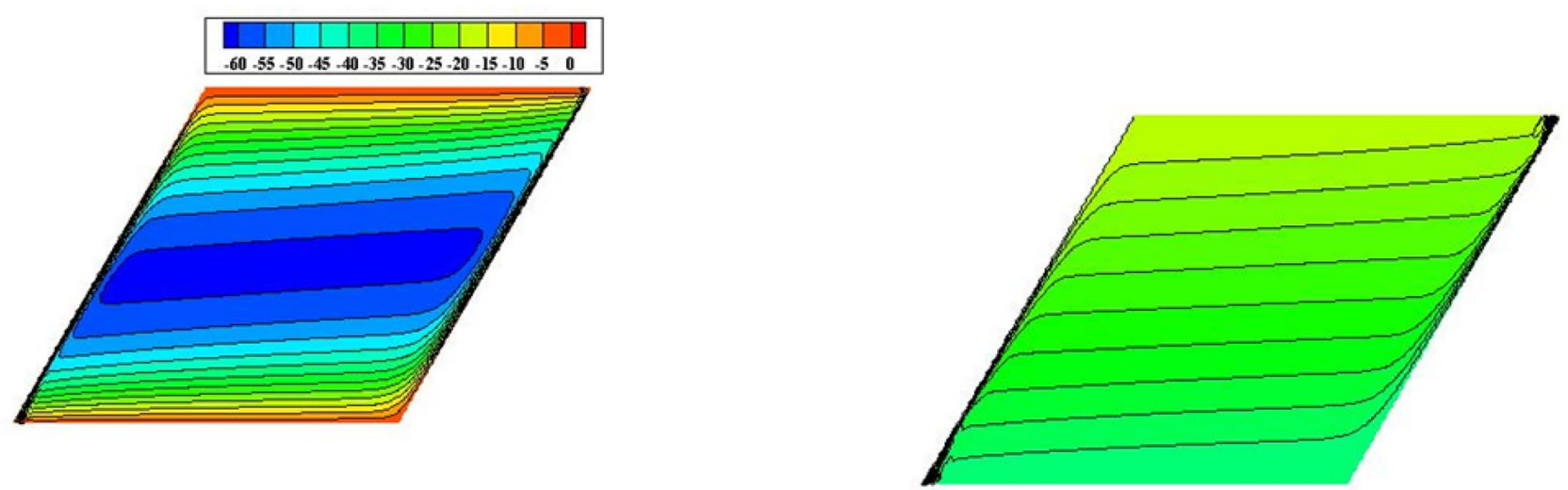

(b) $\alpha=0.9, \beta=0.9$
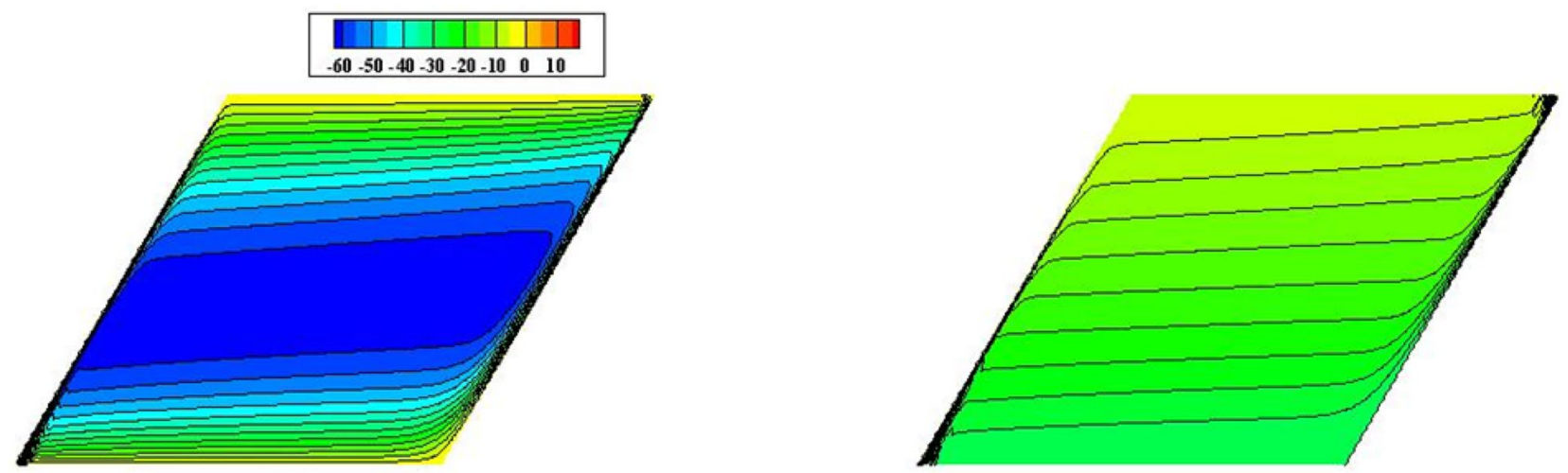

(c) $\alpha=0.8, \beta=0.8$

Fig. 9 Streamlines and isotherms for various values of $a$ and $\beta$ at $A=1, \Phi=30^{\circ}, R a=10^{4}$ 

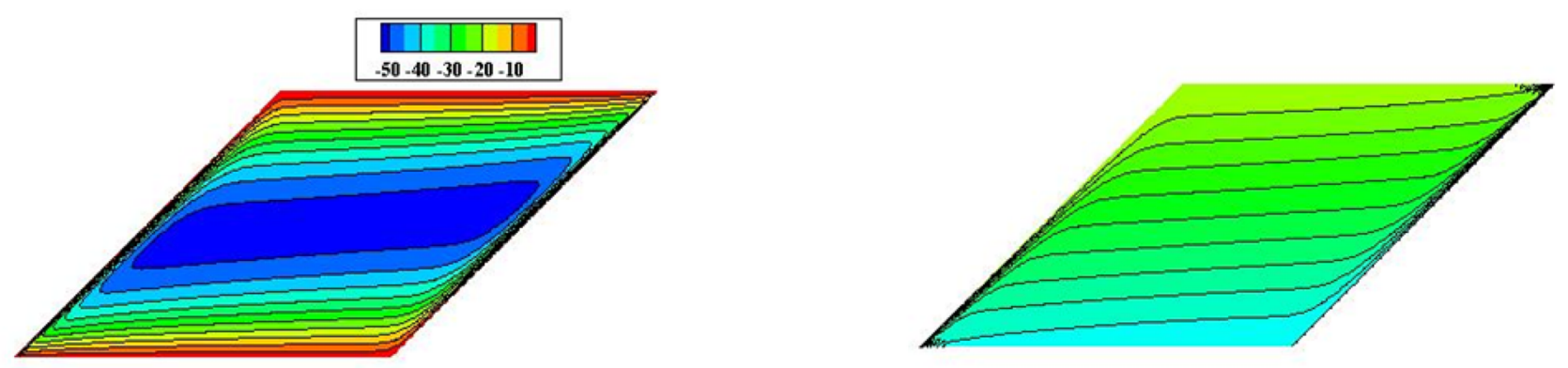

(a) $\alpha=1, \beta=1$
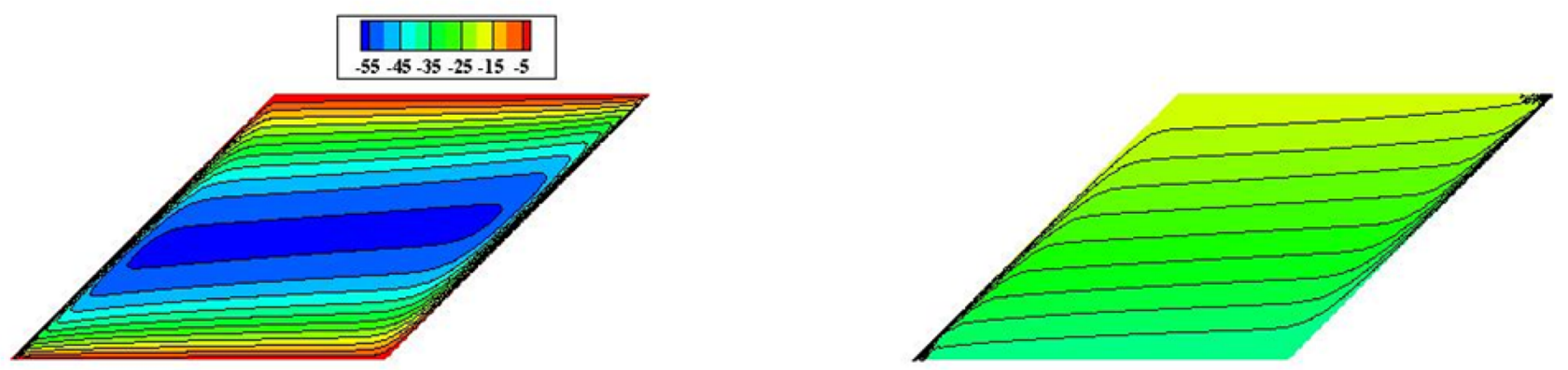

(b) $\alpha=0.9, \beta=0.9$
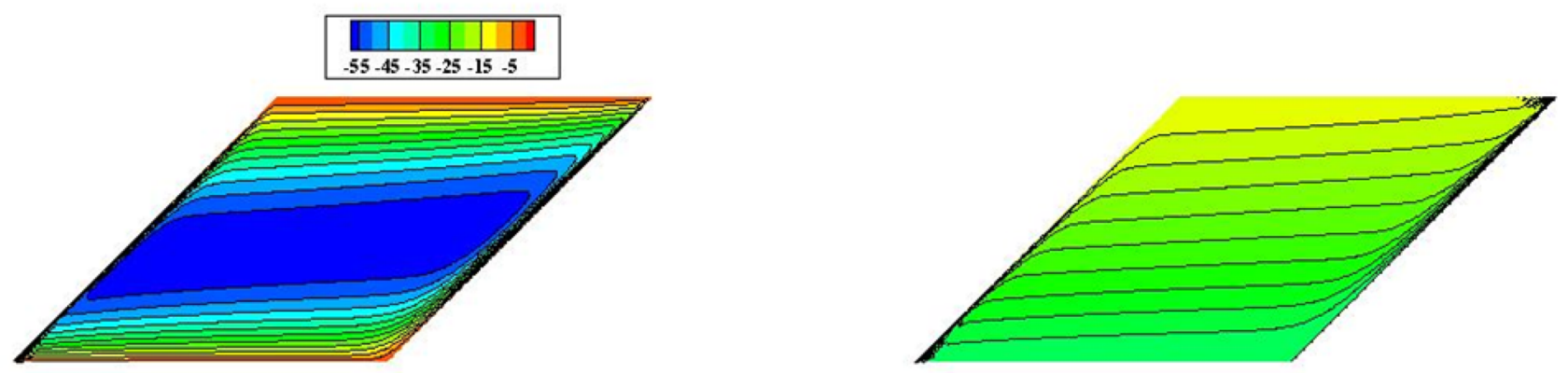

(c) $\alpha=0.8, \beta=0.8$

Fig. 10 Streamlines and isotherms for various values of $a$ and $\beta$ at $A=1, \Phi=45^{\circ}, \operatorname{Ra}=10^{4}$

\section{Conclusion}

The unsteady fluid flow and free convection in a slanted cavity containing a porous medium has been discussed under effect of the conformable fractional derivative. The finite differences method is used to solve the conformable fractional partial differential equations. In the ordinary case at $\alpha$ and $\beta$ equal 1 , the results very agree with the previous result from the open literatures. The following conclusions are summarized from the current study: 


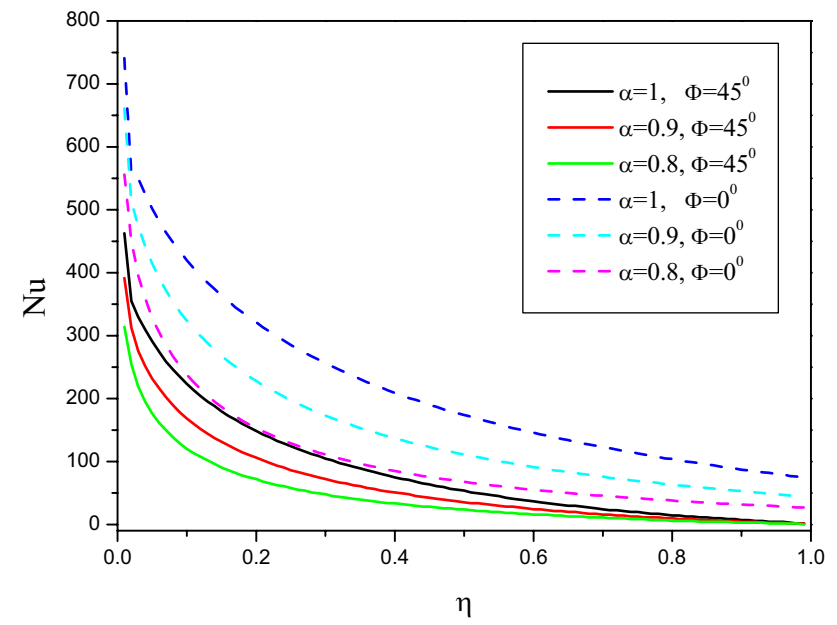

Fig. 11 Nusselt number at the hot wall for various values of $a$ and $\Phi$ at $\mathrm{Ra}=10^{4}$

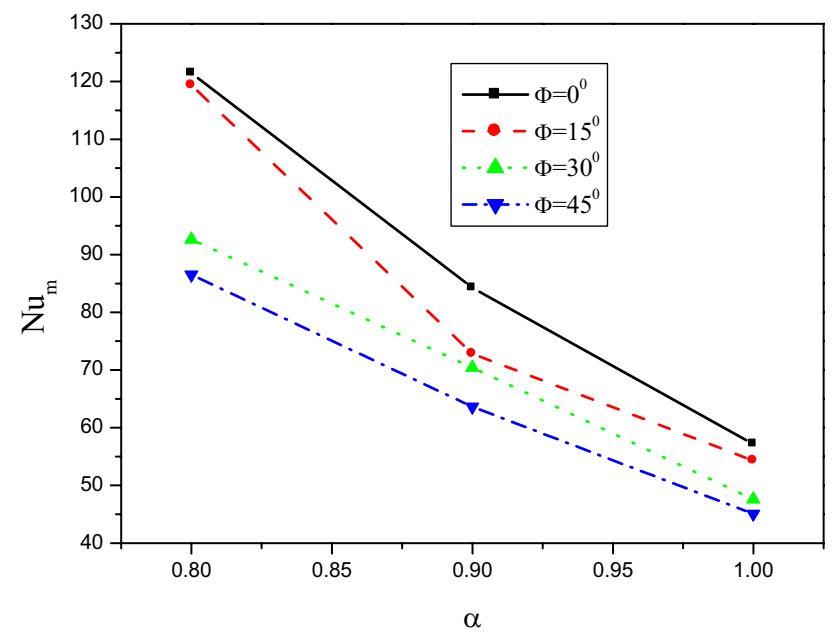

Fig. 12 Average Nusselt number at the hot wall for various values of $a$ and $\Phi$ at $R a=10^{4}$

- In both cases of the low Rayleigh number and high Rayleigh number, values of the fractional derivatives orders less than 1 enhances the flow rate while they reduce rate of the heat transfer.

- The natural convection in case of the high Rayleigh number is the best comparing by the low Rayleigh number.

- The increase in the walls inclination angle gives a clear reduction in both the fluid flow and heat transfer.

Acknowledgements The authors would like to express their gratitude to King Khalid University, Saudi Arabia, for providing administrative and technical support.

\section{Compliance with ethical standards}

Conflict of interest The authors declare that they have no competing interests.

\section{References}

1. Nield DA, Bejan A (1992) Convection in porous media. Springer, New York

2. Van Dam RL, Simmons CT, Hyndman DW, Wood WW (2009) Natural free convection in porous media: first field documentation in groundwater. Geophys Res Lett. https://doi. org/10.1029/2008GL036906

3. Baytas AC, Pop I (1999) Free convection in oblique enclosures filled with a porous medium. Int J Heat Mass Transf 42:1047-1057

4. Baytas AC, Pop I (2001) Free convection in a square porous cavity using a thermal non equilibrium model. Int J Therm Sci 41:861-870

5. Ingham DB, Pop I (1998) Transport phenomenon in porous media. Pergamon, New York

6. Vafai K (1984) Convection flow and heat transfer in variable porosity media. J Fluid Mech 147:233-259

7. Varol Y, Oztop HF, Varo A (2008) Free convection heat transfer and flow field in triangular enclosures filled with porous media. J Porous Media 11(1):103-115

8. Mansour MA, Ahmed SE (2015) A numerical study on natural convection in porous media-filled an inclined triangular enclosure with heat sources using nanofluid in the presence of heat generation effect. Eng Sci Technol Int J 18(3):485-495

9. Mansour MA, Chamkha AJ, Mohamed RA, Abd El-Aziz MM, Ahmed SE (2010) MHD natural convection in an inclined cavity filled with a fluid saturated porous medium with heat source in the solid phase. Nonlinear Anal Model Control 15(1):55-70

10. Mansour MA, Abd El-Aziz MM, Mohamed RA, Ahmed SE (2011) Unsteady natural convection, heat and mass transfer in inclined triangular porous enclosures in the presence of heat source or sink: effect of sinusoidal variation of boundary conditions. Transp Porous Media 87(1):7-23

11. Zhou L, Selim HM (2003) Application of the fractional advection-dispersion equation in porous media. Soil Sci Soc Am J 67:1079-1084

12. Kilbas AA, Srivasava HM, Trujillo JJ (2006) Theory and applications of fractional differential equations. Elsevier, Amsterdam

13. Kilbas A, Srivastava MH, Trujillo JJ (2006) Theory and application of fractional differential equations. North Holland mathematics studies, vol 204. Elsevier, New York

14. Miller K, Ross B (1993) An introduction to the fractional calculus and fractional differential equations. Wiley, New York

15. Kisela T (2008) Fractional differential equations and their applications. Faculty of Mechanical Engineering, Institute of Mathematics, Brno

16. Samko SG, Kibas AA, Marichev OL (1993) Fractional integrals and derivatives: theory and applications. Gordon and Breach, Yverdon

17. Podlubny I (1998) Fractional differential equations: an introduction to fractional derivatives, fractional differential equations, to methods of their solution and some of their applications, vol 198, 1st edn. Elsevier, Amsterdam

18. Abu Hammad I, Khalil R (2014) Legendre fractional differential equation and Legendre fractional polynomials. Int J Appl Math Res 3(3):214-219 
19. Abdeljawad T (2015) On conformable fractional calculus. J Comput Appl Math 279:57-66

20. Karayer H, Demirhan D, Buyukkilic F (2016) Conformable fractional Nikiforov-Uvarov method. Commun Theor Phys 66(1):12-18

21. Zhao D-F, You X-X, Cheng J (2016) Remarks on conformable fractional derivative on time scales. Adv Theor Appl Math 11:61-68

22. Ünal E, Gokdogan A, Çelik E (2015) Solutions of sequential conformable fractional differential equations around an ordinary point and conformable fractional hermite differential equation. arXiv preprint arXiv:1503.05407

23. AbuHammad I, Khalil R (2014) Fractional Fourier series with applications. Am J Comput Appl Math 4(6):187-191

24. Cenesiz Y, Kurt A (2015) The solutions of time and space conformable fractional heat equations with conformable Fourier transform. Acta Univ Sapientiae Math 7(2):130-140

25. Jena RM, Chakraverty S (2019) Solving time-fractional NavierStokes equations using homotopy perturbation Elzaki transform. SN Appl Sci 1(1):16

26. Jena RM, Chakraverty S, Jena SK (2019) Dynamic response analysis of fractionally damped beams subjected to external loads using homotopy analysis method. J Appl Comput Mech 5(2):355-366

27. Jena RM, Chakraverty S (2019) A new iterative method based solution for fractional Black-Scholes option pricing equations (BSOPE). SN Appl Sci 1(1):95

28. Jena RM, Chakraverty S (2019) Residual power series method for solving time-fractional model of vibration equation of large membranes. J Appl Comput Mech 5(4):603-615
29. Liu CY, Guerra AC (1985) Free convection in a porous medium near the corner of arbitrary angle formed by two vertical plates. Int Commun Heat Mass Transf 12(4):431-440

30. Chorin AJ (1967) A numerical method for solving incompressible viscous flow problems. J Comput Phys 2(1):12-26

31. Manole DM, Lage JL (1992) Numerical benchmark results for natural convection in a porous medium cavity. In: Heat and mass transfer in porous media, ASME conference, pp 55-60

32. Li C, Zeng $F$ (2012) Finite difference methods for fractional differential equations. Int J Bifurc Chaos 22(04):1230014

33. Liu F, Yang C, Burrag K (2009) Numerical method and analytical technique of the modified anomalous sub diffusion equation with a nonlinear source term. J Comput Appl Math 231:160-176

34. Lynch VE, Carreras BA, del-Castillo-Negrete D, Ferreira-Mejias KM, Hicks HR (2003) Numerical methods for the solution of partial differential equations of fractional order. J Comput Phys 192:406-442

35. Walker KL, Homsy GM (1978) Convection in a porous cavity. J Fluid Mech 87(3):449-474

36. Bejan A (1979) On the boundary layer regime in a vertical enclosure filled with a porous medium. Lett Heat Mass Transf 6(2):93-102

37. Gross RJ, Bear MR, Hickox CE (1986) The application of flux-corrected transport (FCT) to high Rayleigh number natural convection in a porous medium. In: Proceedings of 8th international heat transfer conference, San Francisco, CA

Publisher's Note Springer Nature remains neutral with regard to jurisdictional claims in published maps and institutional affiliations. 